\title{
A theory of ionospheric thermal radiation
}

\author{
H. C. HsIeH \\ Electron Physics Laboratory, Department of Electrical Engineering, \\ The University of Michigan, Ann Arbor, Michigan, U.S.A.
}

(Received 20 October 1965, revised 30 December 1965)

\begin{abstract}
The ionosphere is considered as a dissipative medium in which the random thermal motions of the charged particles act as a source of thermal radiation. Attention has been focused on the electrons colliding with ions and neutral particles in the ionosphere. A method of analysis has been developed with the aid of the Maxwell and Langevin equations based on a linear, macroscopic, fluctuating electromagnetic field theory. The spectral density of the randomcurrent source function is derived in terms of the conductivity tensor of the ionosphere.

The ionosphere is divided into a large number of incremental volume elements, each containing an ionized medium which represents an anisotropic elementary radiating system, characterized by the spectral density of the source function. The radiation characteristic of the radiating system observed at a point loeated outside of the source region is obtained with the aid of the potential functions which relate the thermal electromagnetic fields at the observation point to their source function. Based on the superposition principle, general expressions have been derived for $w_{0}$, the thermal noise power generated per unit volume, per unit bandwidth, from any given source region $V_{s}$ of the ionosphere, and for $P_{0}\left(f, V_{s}\right)$, the available thermal noise per unit bandwidth at a receiving antenna. These expressions are valid for most regions of interest in the ionosphere where the electron collision process plays a major role in the thermal radiation and they are not limited in frequency range.
\end{abstract}

\section{INTRODUCTION}

IT Is well known that because the ionosphere acts as an absorber of radio waves, it can also act as an emitter of thermal radio noise. It has been conclusively demonstrated by various workers (PAwsey et al., 1951; GARDNER, 1954; Dowden, 1960; Lrrte et al., 1961) that the thermal emission from the $D$-region can, under favorable conditions, be observed with a dipole antenna. For example, Pawsey et al. (1951) have identified and measured the thermal radiation from the ionosphere in the vicinity of $2 \mathrm{Mc} / \mathrm{s}$ in the temperate latitude.

It appears that usually the thermal radiation has been neglected because its level is exceedingly low as illustrated by PAwser et al. (1951) and it does not constitute an appreciable source of interference in radio communication. However, the noise radiated from a plasma (e.g. the ionosphere) is not necessarily a detrimental effect in all cases, as it is in communication, since if the spectral distribution of the emitted energy is characteristic of the plasma properties, a measurement of radiation provides specific information on the plasma. For example, knowledge of the radiated power gives a measure of the electron temperature in the plasma and this has been used as a powerful diagnostie technique.

It is well known that the thermal radiation from dissipative bodies is due to the random thermal motion of the charges in the body. If the body is at a uniform temperature, one approach that may be used for studying radiation may be called 
the integral approach. The body as a whole is considered to be nonradiating and the power that is absorbed from its surroundings, which is assumed to be at the temperature of the body, can be computed. This power is set equal to the power radiated by the body. In this approach no attempt is made to determine the noise current fluctuations that are the cause of the thermal radiation. In those cases in which the temperature of the body is nonuniform this approach fails.

Another approach, which may be called the 'Nyquist source treatment' (RYTov, 1959; Hats, 1961; Vanwormhoudt and Hads, 1962), focuses attention upon the sources of the radiation and determines their relevant statistical properties. Once these are known, the determination of the radiation is conceptually a simple problem, although mathematical difficulties usually arise.

In the present study, the 'Nyquist source treatment' is adopted and the ionosphere is considered as an anisotropic dissipative medium in which the random thermal motions of the charged particles act as a source of the thermal radiation. It is further postulated that in the ionosphere a linear constitutive local relation exists between the driven a.c. conduction current density $\mathbf{J}_{d}$ and an applied a.e. electric field intensity $\mathbf{E}$ of the form

$$
\mathbf{J}_{\partial}(\omega, \mathbf{r})=\boldsymbol{\sigma}(\omega, \mathbf{r}) \cdot \mathbf{E}(\omega, \mathbf{r}),
$$

where $\boldsymbol{\sigma}$ is the conductivity tensor of the ionosphere, and a function of the angular frequency $(\omega)$ and position variable $\mathbf{r}$ which characterize the medium under consideration. A small-signal analysis is made throughout the present paper.

\section{Derryation of the Conductivity Tensor}

For a macroscopic analysis the Langevin equation can be used effectively to describe the motion of an electron, and it can be expressed as follows:

$$
\left.m \frac{\partial \mathbf{v}}{\partial t}+m \nu \mathbf{v}=e \mid \mathbf{E}+\mathbf{v} \times \mathbf{B}\right\rfloor
$$

where $\mathbf{B}(\mathbf{r})$ is the static geomagnetic field, $v(r)$ is the average electronic collision frequency with ions and neutral particles, $e, m$ and $\mathbf{v}$ are the electronic charge taken as a negative value, mass and velocity respectively.

On the other hand the convection density $\mathbf{J}$ is related to the velocity $\mathbf{v}$ by

$$
\mathbf{J}=N_{0} e \mathbf{v},
$$

where $N_{0}(\mathbf{r})$ is the electron number density.

Assuming the time harmonic variation $e^{j \omega t}$ for the quantities of interest, upon elimination of $\mathbf{v}$ from equations (2) and (3) the following relationship is established:

where

$$
U \mathbf{J}+j(\mathbf{J} \times \mathbf{Y})=-j \omega \varepsilon_{0} X \mathbf{E},
$$

$$
\begin{array}{ll}
X=\frac{\omega_{n}^{2}}{\omega^{2}}, & \omega_{p}^{2}=\frac{N_{0} e^{2}}{m \varepsilon_{0}} \\
\mathbf{Y}=\frac{e \mathbf{B}}{m \omega}, & Y=\frac{\omega_{b}}{\omega}=\begin{array}{c}
-e|\mathbf{B}| \\
m \omega
\end{array}, \\
Z=\frac{\nu}{\omega}, & U=1-j Z,
\end{array}
$$


in which $\omega_{p}$ and $\omega_{b}$ are the plasma and gyrofrequencies of the electrons, respectively, and $\varepsilon_{0}$ is the dielectric constant of vacuum.

On the other hand, the geomagnetic field $\mathbf{B}$ can be approximated by a dipole field which is induced by a uniformly magnetized spherical Earth, and may be expressed (MoRGAN, 1959) as

$$
\mathbf{B}=\frac{M a^{3}}{3} \nabla\left(\frac{\cos \theta}{r^{2}}\right),
$$

where the space variables $r$ and $\theta$ denote, respectively, the radial and polar angular coordinates of the geomagnetic spherical coordinate system with its origin located at the center of the Earth, and the constants $M$ and $a$ are the magnetization and the radius of the Earth respectively. By adopting this model of the geomagnetic field, the $r$-component $Y_{1}$, the $\theta$-component $Y_{2}$ and the $\varphi$-component $Y_{3}$ of the vector $\mathbf{Y}$ are given by

where

$$
Y_{1}=2 G \cos \theta, \quad Y_{2}=G \sin \theta \quad \text { and } \quad Y_{3}=0,
$$

$$
G=\left(\frac{-e}{\omega m}\right) \frac{M}{3}\left(\frac{a}{r}\right)^{3} .
$$

Furthermore, by writing a vector as a column matrix the vector equation (4) may be conveniently expressed in the following matrix form:

or equivalently in tensor notation as $\underline{\gamma \mathrm{J}}=\underline{\mathrm{E}}$

$$
\boldsymbol{\gamma} \cdot \mathbf{J}=\mathbf{E}
$$

where the resistivity matrix $\gamma$ is defined as

$$
\stackrel{=}{\gamma}=\left\{\gamma_{\alpha \beta}\right\}, \quad \alpha, \beta=1,2,3,
$$

with its elements being given by

$$
\begin{aligned}
& \gamma_{11}=\gamma_{22}=\gamma_{33}=\frac{j U}{\omega \varepsilon_{0} X}, \\
& \gamma_{12}=-\gamma_{21}=0, \\
& \gamma_{13}=-\gamma_{31}=\frac{G \sin \theta}{\omega \varepsilon_{0} X}, \\
& \gamma_{23}=-\gamma_{32}=\frac{-2 G \cos \theta}{\omega \varepsilon_{0} X},
\end{aligned}
$$

and with its determinant $|\underline{\underline{\gamma}}|$ given by

in which

$$
\stackrel{|\gamma|}{=}=\frac{j U}{\left(\omega \varepsilon_{0} X\right)^{3}}\left[Y^{2}-U^{2}\right]
$$

$$
Y^{2}-G^{2}\left(1+3 \cos ^{2} \theta\right) .
$$

$\underset{|\gamma|}{=}$ can be zero only for a special situation where $\nu=0$ and $\omega=\omega_{b}$ occur simultaneously. Since $v=0$ is not of interest to the present study, $\underset{=}{|\gamma|}$ can be considered 
to possess an inverse, which is denoted by $\underset{\underline{\sigma}}{\underline{a}}$ and is referred to as the 'conductivity matrix', i.e.,

$$
\underline{\underline{\sigma}}=\underline{\underline{I}}=
$$

where $I$ is the unit matrix. Consequently, from equations $9 a$ and $14, \underline{J}$ can be expressed in terms of $\underline{\mathbf{E}}$ explicitly as

$$
\underline{J}=\underline{\sigma} \underline{E}
$$

or in a tensor notation as

$$
\mathbf{J}=\boldsymbol{\sigma} \cdot \mathbf{E},
$$

where

$$
\sigma_{\alpha \beta}=D C_{\alpha \beta}, \quad \alpha, \beta=1,2,3,
$$

where

$$
D=j \omega \varepsilon_{0} \frac{X}{U\left(U^{2}-Y^{2}\right)}
$$

with

$$
\begin{aligned}
& C_{11}=\left(Z^{2}-1+4 G^{2} \cos ^{2} \theta\right)+j 2 Z \\
& C_{22}=\left(Z^{2}-1+G^{2} \sin ^{2} \theta\right)+j 2 Z \\
& C_{33}=\left(Z^{2}-1\right)+j 2 Z \\
& C_{12}=C_{21}=2 G^{2} \sin \theta \cos \theta \\
& C_{13}=-C_{31}=-(Z+j) C \sin \theta \\
& C_{23}=-C_{32}=2(Z+j) G \cos \theta .
\end{aligned}
$$

\section{Notse Power Radiated from the Ionosphere}

A body with a non-uniform temperature distribution is not in the thermodynamic equilibrium. However, in those cases in which the distribution function of charge carriers deviates only slightly from the equilibrium distribution (so as to produce heat and current flow), and this includes all cases for which a temperature can be reasonably defined, it would be expected that the radiated noise power could still be computed as the superposition of the noise power radiated from the various volume elements of the body. In this case each element at a particular temperature radiates the same noise power it would radiate at equilibrium at the same temperature. Such an analysis calls for an approach to the fluctuation problem that considers each differential volume element separately as an absorber and emitter of noise power. It calls for the introduction of a source term into Maxwell's equations analogous to the source term of the Langevin equation in the theory of Brownian motion.

Although Maxwell's equations and the constitutive relation are sufficient to solve most electromagnetic problems, they are insufficient for noise studies. The current density derived from the constitutive relation represents only the current driven by the electromagnetic fields. Besides this driven current, the current density fluctuation caused by the random motion of the charge must be considered. This 
can be taken into account by introducing into Maxwell's equations a random driving current density distribution which is independent of the electromagnetic fields, i.e.

$$
\nabla \times \mathbf{e}=-\mu_{0} \frac{\partial \mathbf{h}}{\partial t}
$$

and

$$
\nabla \times \mathbf{h}=\varepsilon_{0} \frac{\partial \mathbf{e}}{\partial t}+\mathbf{i}
$$

where $e$ and $\mathbf{h}$ are the time-dependent electric and magnetic fields, respectively, and $i$ is the current density, $\mu_{0}$ is the permeability of vacuo. The current density $i$ in equation 19 consists of two parts. First of all there is the 'driven' component $i_{d}$ that is produced by the electric field $e$ and is related to $e$ by equation 15 . The spontaneous noise fluctuations of the field at thermal equilibrium can be taken into account by another current component of $i$ in equation 19 , the source current density $\mathbf{K}(t, \mathbf{r})$, a statistical quantity which is a stationary function of time.

\subsection{The dyadic spectral density of current source functions}

In the study of problems involving radiation of noise power, it is convenient to introduce Fourier transformations in time of all field quantities in equations 18 and 19. In the present case all random time functions are stationary and, strictly speaking, they do not possess Fourier transformations. However, this difficulty may be overcome by constructing a periodic substitute function (RYTov, 1959; Haus, 1961) according to the definition

and

$$
\mathbf{F}(t, \mathbf{r}, T)=\mathbf{F}(t, \mathbf{r}), \quad \text { for } \quad-\frac{T}{2}<t<\frac{T}{2}
$$

$$
\mathbf{F}(t+n T, \mathbf{r}, T)=\mathbf{F}(t, \mathbf{r}, T)
$$

These substitute functions have Fourier transformations of the form

$$
\mathbf{F}(\omega, \mathbf{r}, T)=\frac{1}{T} \int_{-T / 2}^{T / 2} \mathbf{F}(t, \mathbf{r}, T) e^{-j \omega t} d t
$$

In the limit as $T \rightarrow \infty$, the substitute functions are indistinguishable from their originals. The spectral density of any noise process can be obtained directly from the ensemble average of products of these Fourier components. Thus, the dyadic spectral density of $\mathbf{F}$ is given by

$$
\mathbf{S}_{\mathbf{F}}\left(\omega, \mathbf{r}, \mathbf{r}^{\prime}\right)=\lim _{T \rightarrow \infty} \frac{T}{2 \pi}\left\langle\mathbf{F}(\omega, \mathbf{r}, T) \mathbf{F}^{*}\left(\omega, \mathbf{r}^{\prime}, T\right)\right\rangle_{\text {avg }},
$$

where the symbol $*$ denotes the complex conjugate.

It should be noted that the spectral analysis of the periodic substitute function leads to a discrete spectrum extending over negative, as well as positive, frequencies. With lines at frequenoy interval $\Delta f=(1 / T)$ the expression

$$
\left\langle 2 \mathbf{F}(\omega, \mathbf{r}, T) \mathbf{F}^{*}\left(\omega, \mathbf{r}^{\prime}, T\right)\right\rangle_{\text {avg }}=4 \pi \Delta f \mathbf{S}_{\mathbf{r}}\left(\omega, \mathbf{r}, \mathbf{r}^{\prime}\right) .
$$

may be identified in the limit of large $T$ as 'the mean-square fluctuation of $\mathbf{F}$ ' in 
the frequency interval $\Delta f$, Furthermore, for a stationary time function $\mathbf{F}$ (Blanc-Lapierse and Fortet, 1953),

$$
\frac{T}{2 \pi}\left\langle\mathbf{F}(\omega, \mathbf{r}, T) \mathbf{F}^{*}\left(\omega^{\prime}, \mathbf{r}, T\right)\right\rangle_{\mathrm{avg}}=0, \quad \omega \neq \omega^{\prime} .
$$

Precisely this kind of treatment must be kept in mind in applying the formal expansion of the Fourier integral and using the spectral amplitude densities in the study of electromagnetic fluctuation on which the present paper is based.

As a matter of convenience, for a particular physical variable, the lower case letter is used for the stationary time function and for its periodic substitute function, while the capital letter is used for its Fourier transform in the following discussion. For example, it is obvious, from equations (18) and (19), with the aid of equation (15b) that the Fourier amplitude of the periodic substitute functions is related in the following manner:

and

$$
\nabla \times \mathbf{E}=-j \omega \mu \mathbf{H}
$$

$$
\nabla \times \mathbf{H}=j \omega \varepsilon_{0} \mathbf{E}+\boldsymbol{\sigma} \cdot \mathbf{E}+\mathbf{K} .
$$

Suppose that a region of the ionosphere under study is divided into a large number of sufficiently small elementary volume elements such that within each one of these elementary volumes the medium may reasonably be assumed to be uniform at a certain temperature $T_{0}$. Strictly speaking these elementary volume elements should be made to approach zero. On the other hand, they have to be kept large enough to contain a large number of charge carriers in order that statistical arguments may be applicd. A tensor-conductivity description of the medium as given by equation (15b) is possible only because the current in an elementary volume depends upon the electric field in the same volume, but not upon its derivatives, that is, upon the value of the electric field in the neighboring elementary volumes. In view of this fact, it is quite reasonable to expect that the source current caused by the random motion of the charge carriers in two neighboring elementary volumes are uncorrelated. In other words, if $\mathbf{r}$ and $\mathbf{r}^{\prime}$ denote the points belonging to two different elementary volumes, then $\mathbf{K}(\omega, \mathbf{r})$ and $\mathbf{K}\left(\omega, \mathbf{r}^{\prime}\right)$ are not correlated and the dyadic spectral density of $\mathbf{K}$ has the form

$$
\mathbf{S}_{\mathbf{K}}\left(\omega, \mathbf{r}, \mathbf{r}^{\prime}\right)=\delta\left(\mathbf{r}-\mathbf{r}^{\prime}\right) \psi(\omega, r),
$$

where $\delta\left(\mathbf{r}-\mathbf{r}^{\prime}\right)$ is the usual Dirac delta function.

On the other hand, an elementary volume element may be considered as a linear network containing a noise source in thermal equilibrium and the technique developed in the theory of linear noise networks (HaUS, 1961; VaNwormmoudT and HaUs, 1962), which makes use of the generalized Nyquist theorem, can be applied. Using the concept of a linear network, for example, Hats (1961) has obtained a simple expression for $\psi(\omega, \mathbf{r})$ as follows:

$$
\psi(\omega, \mathbf{r})=\frac{k T_{0}(r)}{2 \pi}[\boldsymbol{\sigma}(\omega, \mathbf{r})+\boldsymbol{\sigma} \dagger(\omega, \mathbf{r})],
$$

where $k$ is the Boltzmann constant and the symbol dagger $(\dagger)$ indicates the complexconjugate transpose of the conductivity matrix $\sigma$. If the average volume density of 
thermal energy $\tau(r)$ in $\mathrm{J} / \mathrm{m}^{3}$ is introduced, defined as the ratio of the amount of thermal energy generated within an elementary volume $\Delta V$ to the volume $\Delta V$, then from equations (27) and (28) one has

$$
\mathbf{S}_{K}(\omega, \mathbf{r})=\frac{\tau(r)}{2 \pi}[\boldsymbol{\sigma}(\omega, \mathbf{r})+\boldsymbol{\sigma} \dagger(\omega, \mathbf{r})]
$$

and from equation (23)

$$
\left\langle 2 \mathbf{K}(\omega, \mathbf{r}) \mathbf{K}^{*}(\omega, r)\right\rangle_{\text {avg }}=2 \Delta f \tau(r)[\boldsymbol{\sigma}(\omega, \mathbf{r})+\boldsymbol{\sigma} \nmid(\omega, \mathbf{r})],
$$

which may be given alternatively in its component form with the aid of equation (17) as follows:

where

$$
\left\langle 2 K_{\alpha}(\omega, r) K_{\beta}^{*}(\omega, r)\right\rangle_{\mathrm{avg}}=\tau(r) \Delta f L_{\alpha \beta}(\omega, r),
$$

with

$$
L_{\alpha \beta}(\omega, r)=4 \omega \varepsilon_{0}\left(\frac{X Z}{1+Z^{2}}\right)\left[l_{\alpha \beta}+j m_{\alpha \beta}\right], \quad \alpha, \beta=1,2,3,
$$

$$
\begin{aligned}
l_{11} & =\frac{1}{Q(Y, Z)}\left[\left(1+Z^{2}\right)\left(1+Z^{2}+Y^{2}\right)+\left(Z^{2}+Y^{2}-3\right) 4 G^{2} \cos ^{2} \theta\right], \\
l_{22} & =\frac{1}{Q(Y, Z)}\left[\left(1+Z^{2}\right)\left(1+Z^{2}+Y^{2}\right)+\left(Z^{2}+Y^{2}-3\right) G^{2} \sin ^{2} \theta\right], \\
l_{33} & =\frac{1}{Q(Y, Z)}\left[\left(1+Z^{2}\right)\left(1+Z^{2}+Y^{2}\right)\right], \\
l_{12} & =l_{21}=\frac{1}{Q(Y, Z)}\left[\left(Z^{2}+Y^{2}-3\right) G^{2} \sin 2 \theta\right], \\
l_{13} & =l_{31}=l_{23}=l_{32}=0, \\
m_{11} & =m_{22}=m_{33}=m_{12}=m_{21}=0, \\
m_{13} & =-m_{31}=\frac{1}{Q(Y, Z)}\left[2\left(1+Z^{2}\right) G \sin \theta\right], \\
m_{23} & =-m_{32}=\frac{-1}{Q(Y, Z)}\left[4\left(1+Z^{2}\right) G \cos \theta\right]
\end{aligned}
$$

and

$$
Q(Y, Z)=\left(Y^{2}+Z^{2}-1\right)^{2}+4 Z^{2} .
$$

It is observed that $Y=0$ when $G=0$. In this case, $l_{\alpha \beta}=1$ if $\alpha=\beta$ and $l_{\alpha \beta}=0$ if $\alpha \neq \beta$, while $m_{\alpha \beta}$ becomes zero regardless of whether $\alpha=\beta$ or $\alpha \neq \beta$. This suggests that the tensor $\left\{L_{\alpha \beta}\right\}$ appearing in equation (31) becomes a scalar and the medium becomes isotropic. This is perfectly reasonable since when $G=0$ the geomagnetic field is completely absent.

It is also interesting to note that for the case

$$
Y^{2}+Z^{2}=3
$$

$l_{\alpha \beta}$ again becomes either equal to unity or to zero according to whether $\alpha=\beta$ or $\alpha \neq \beta$ and

$$
m_{13}=-m_{31}=\frac{1}{2} G \sin \theta
$$


and

$$
m_{23}=-m_{32}=-G \cos \theta .
$$

\subsection{Time average thermal noise power radiated}

In view of the fact that for the periodic field the average time rate of change of stored energy is zero, the total average power radiated from a system of a current may be given by (STRATTON, 1941)

$$
W=\oint_{S} \overline{\mathbf{p}} \cdot d \mathbf{S}=-\frac{1}{2} \operatorname{Re} \int_{V}\left(\mathbf{E} \cdot \mathbf{I}^{*}\right) d V,
$$

where $\mathbf{p}$ is the Poynting vector. Thus radiation can be calculated either by integrating the normal component of the Poynting vector over a closed surface $S$ including all sources or by integrating the power expended per unit volume over the current distribution. In the present discussion the latter approach is taken.

Keeping in mind that the concern here is with the random current distribution and since the time average power radiated per unit volume, $u(\omega, \mathbf{r})$, is given by

$$
w(\omega, \mathbf{r})=\frac{1}{2} \operatorname{Re}\left[\mathbf{K}^{*} \cdot \mathbf{E}\right], \quad \mathrm{W} / \mathbf{m}^{3},
$$

in which $\mathbf{K}$ is the cause and $\mathbf{E}$ is its effect, and with the aid of equation $(9), w(\omega, \mathbf{r})$ becomes

$$
w(\omega, \mathbf{r})=\frac{1}{2} \operatorname{Re}\left[\mathbf{K}^{*} \cdot(\boldsymbol{\gamma} \cdot \mathbf{K})\right]=\frac{1}{2} \operatorname{Re}[\underline{\mathbf{K}} \underline{\underline{\gamma}} \underline{\underline{\mathbf{K}}}] .
$$

The substitution of equation (11) into equation (38) yields

$$
w(\omega, r)=\frac{Z}{2 \omega \varepsilon_{0} X}\left[K_{1} K_{1}{ }^{*}+K_{2} K_{2}{ }^{*}+K_{3} K_{3}{ }^{*}\right] .
$$

On the other hand, with the aid of equations (31) and (32), the thermal noise power generated per unit volume, per unit bandwidth, $w_{0}(f, r)$, may be given as

$$
w_{0}(f, \mathbf{r})=k T_{0}\left(\frac{Z^{2}}{1+Z^{2}}\right)\left[l_{11}+l_{22}+l_{33}\right]
$$

where $l_{11}, l_{22}$ and $l_{33}$ are given in equation (33).

It is interesting to observe that $w_{0}$ given in equation (40) does not depend explicitly upon the electron number density $N_{0}$ since it does not contain the parameter $X$.

\section{Observation of Thermal Radiation from the Ionosphere}

The rigorous determination of the radiation intensity within the emitting region of the ionosphere must be based on the study of the electromagnetic wave propagation in an anisotropic absorbing medium, in which each volume element can act as an emitter as well as an absorber of the thermal radiation. However, this problem is not discussed in the present paper.

Nevertheless, it is of interest and of a considerable practical importance to know about the charactcristics of noise power roceivod from the ionospherie thermal radiation at a detecting antenna located outside of the source region.

In view of the fact that the relation of the radiation fields to their sources is most readily found in terms of potential functions, and since the information with 
regard to some statistical properties of the random source current function $\mathbf{K}$ is available from Section 3, the retarded vector potential function is introduced here and expressed in complex form as $\mathbf{A}\left(\omega, x_{\alpha}\right) e^{j \omega t}$, with

$$
\mathbf{A}\left(\omega, x_{\alpha}\right)=\frac{\mu_{0}}{4 \pi} \int_{V_{s}} \frac{\mathbf{K}\left(\omega, x_{\alpha}{ }^{\prime}\right) \exp \left(-j k_{0} R\left(x_{\alpha}, x_{\alpha}{ }^{\prime}\right)\right)}{R\left(x_{\alpha}, x_{\alpha}{ }^{\prime}\right)} d V^{\prime},
$$

where $x_{\alpha}$ and $x_{\alpha}{ }^{\prime}$ denote the coordinates of the observation point and the source point respectively, and $R\left(x_{\alpha}, x_{\alpha}{ }^{\prime}\right)$ is the distance between them. $V_{s}\left(x_{\alpha}{ }^{\prime}\right)$ is the volume of the source region under investigation and $k_{0}$ is the wave number. In the present discussion $x_{\alpha}$ is taken in the air and $x_{\alpha}{ }^{\prime}$ is taken in the ionosphere.

It should be observed that equation (41) signifies superposition of the solutions of the inhomogeneous wave equation

$$
\nabla^{2} \mathbf{A}+k_{0}{ }^{2} \mathbf{A}=-\mathbf{K}
$$

where $k_{0}{ }^{2}=\omega^{2} \mu_{0} \varepsilon_{0}$ and corresponds to a source at the point $x_{a}^{\prime}$ given by $\mathbf{K}=$ $C \delta\left(x_{\alpha}-x_{\alpha}{ }^{\prime}\right)$, with $\delta\left(x_{\alpha}-x_{\alpha}{ }^{\prime}\right)$ being the usual Dirac delta function. On the other hand the retarded scalar potential function $\Phi\left(\omega, x_{\alpha}\right)$ is related to the vector potential by (Stratton, 1941)

$$
\nabla \cdot A+j \omega \mu \varepsilon \Phi=0
$$

which expresses the idea of conservation of charge. It should be noted that equation (43) is valid in free space (air) whereas it is only an approximation in a region of the conducting medium in which $|\sigma| j \omega \varepsilon_{0} \mid \ll 1$.

It is well known that the electromagnetic fields at an observation point $x_{\alpha}$, taken in air, can be derived from these potential functions by

and

$$
\mathbf{E}=-\nabla \Phi-j \omega \mathbf{A}
$$

$$
\mathbf{H}=\frac{1}{\mu_{0}} \nabla \times \mathbf{A},
$$

where the spatial differential operator $\nabla$ should be understood as $\nabla_{x_{\alpha}}$, which only operates on the function of $x_{\alpha}$. The utilization of potential functions is particularly convenient because space differentiation $\nabla$, under the sign of the volume integration, does not touch $\mathbf{K}\left(\omega, x_{\alpha}{ }^{\prime}\right)$ and thereby the field intensities $\mathbf{E}$ and $\mathbf{H}$ in the same manner do not contain derivatives of $\mathbf{K}$.

Upon substitution of equations (41) and (43) into equations (44) and (45) and if only a $1 / R$ dependent radiation field is taken into account, the electric and magnetic fields may be written as

and

$$
\mathbf{E}=\frac{1}{j \omega \varepsilon} \frac{1}{4 \pi} \int_{V_{s}}[\mathbf{k} \times(\mathbf{K} \times \mathbf{k})] \frac{e^{-j \mathbf{k} \cdot \mathbf{R}}}{R} d V^{\prime}
$$

$$
\mathbf{H}=\frac{1}{4 \pi} \int_{V_{\mathrm{s}}}(\mathbf{K} \times \mathbf{k}) \frac{e^{-j \mathbf{k} \cdot \mathbf{R}}}{R} d V^{\prime}
$$

in which the propagation vector $\mathbf{k}=\mathbf{n} k_{0}$ is introduced and the unit vector $\mathbf{n}$ is defined as $\mathbf{R} / R$ so that $\mathbf{k}$ and $\mathbf{R}$ are in the same direction. 
The electromagnetic tields given by equations (46) and (47) can be considered as the random thermal electromagnetic fields since their source function $\mathbf{K}$ is a random, statistical quantity. The time average power flow density at the observation point $x_{\alpha}$ may be considered now with the aid of the Povnting vector defined as

$$
\overline{\mathbf{p}}=\frac{1}{2} \operatorname{Re}\left[\mathrm{E} \times \mathrm{H}^{*}\right] .
$$

It is not difficult to show that the substitution of equations (46) and (47) into equation (48) yields

$$
\overline{\mathbf{p}}\left(\omega, x_{\alpha}\right)=\frac{k_{0} \Delta f}{2 \lambda^{2}} \int_{V_{k}} \mathbf{n}\left(x_{\alpha}, x_{\alpha}{ }^{\prime}\right)\left[\begin{array}{c}
k T_{0} X Z \\
1+Z^{2}
\end{array}\right] \frac{\Gamma\left(x_{\alpha}, x_{\alpha}{ }^{\prime}\right)}{R^{2}\left(x_{\alpha}, x_{\alpha}{ }^{\prime}\right)} d V^{\prime},
$$

where $\lambda$ is the free-space wavelength, $Z, X$ and $T_{0}$ are functions of the source point coordinate $x_{\alpha}^{\prime}$ and $\Gamma\left(x_{x}, x_{\alpha}{ }^{\prime}\right)$ is defined by

$$
\Gamma\left(x_{\alpha}, x_{\alpha}{ }^{\prime}\right)=\left(1-n_{1}{ }^{2}\right) l_{11}+\left(1-n_{2}{ }^{2}\right) l_{22}+\left(1-n_{3}{ }^{2}\right) l_{33}-2 n_{1} n_{2} l_{12},
$$

in which $n_{1}, n_{2}$ and $n_{3}$ are the components of the unit vector $\mathbf{n}$ along $\mathbf{r -}, \theta$ - and $\varphi$-coordinate axes, and $l_{11}, l_{22}, l_{33}$ and $l_{12}$ are given in equation (33).

It should be observed that equation (49) is based on the concept that the radiation intensity in any solid angle can be treated as energy, transferable in a bundle of plane, nonextinguishable waves whose normals are included in the solid angle. In a homogeneous isotropic medium the direction of the vector of energy flux coincides with the wave normal (RYTov, 1959). The unit vector $\mathbf{n}\left(x_{\alpha}, x_{\alpha}^{\prime}\right)$ indicates the direction of propagation of the wave orginating at the source point $x_{\alpha}$ '.

Since the time average Poynting vector $\overline{\mathrm{p}}\left(\omega, x_{\alpha}\right)$ is determined, the noise power received from the ionospheric thermal radiation at the receiving antenna can be obtained by taking a proper surface integral of $\bar{p}\left(\omega, x_{\alpha}\right)$ over the aperture of the antenna $A_{0}$,

$$
P(\omega)=\int_{A_{0}} \overline{\mathbf{p}}\left(\omega, x_{\alpha}\right) \cdot \mathbf{d s},
$$

where $\mathbf{d s}=\mathbf{n}_{0} d s$, with $\mathbf{n}_{0}$ being a unit vector normal to the differential surface area $d s$.

It should be noted that $P(\omega)$, given by equation (51) can be regarded as the available noise power at the receiving antenna in the frequency interval between $f$ and $f+\Delta f$. On the other hand, from an elementary antenna theory (KRAUs, 1950 ), if the receiving antenna is properly oriented for maximum response, the available noise power $P(\omega)$ can be given by

$$
P(\omega)=A_{e} p_{0}(\omega)
$$

where $p_{0}(\omega)$ is the time average Poynting vector at the position of the receiving antenna and $A_{e}=\left(\lambda^{2}\right) / \Omega_{a}$ is the effective area of the antenna, with $\Omega_{a}$ being the solid angle through which all of the power radiated would stream if the power per unit solid angle equaled the maximum value of radiation intensity over the beam area.

In order to determine $\mathbf{p}\left(\omega, x_{\alpha}\right)$ from equation (49) the source region $V_{s}$, which is determined by the beam area of the receiving antenna, must be specified and the 
integrand must be expressed as a function of conveniently chosen coordinate variables. Although the parameter $l_{\alpha \beta}$ was expressed in spherical coordinate variables $(r, \theta, p)$ in the previous section it is not difficult to see that the integration can conveniently be introduced with respect to the solid angle, subtended at the observation point, instead of carrying out the volume integration in a spherical coordinate system as in equation (49); this is illustrated in the following discussion.

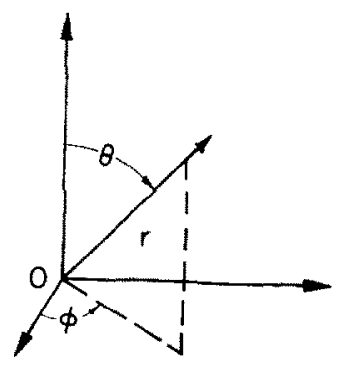

(a)

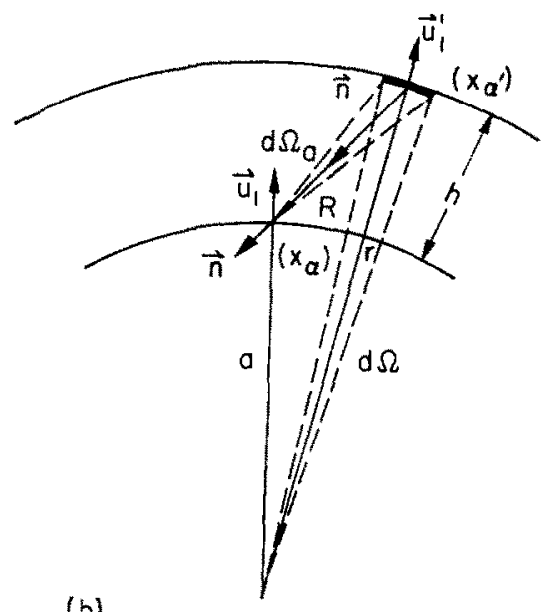

(b)

Fig. 1. Coordinate system and definition of variables. (a) Geomagnotic sphorical coordinate system. (b) Geometrical relation between the source points $x_{\alpha}{ }^{\prime}$ and the observation points $x_{\alpha}$.

If $d \Omega$ and $d \Omega_{a}$ denote, respectively, the differential solid angle subtended at the origin (the center of the Earth) and at the observation point (on the surface of the Earth) by a source located at $x_{\alpha}{ }^{\prime}$, then it is not difficult to see that with the aid of of Fig. 1

$$
\frac{R^{2} d \Omega_{a}}{\left|\left(\mathbf{n} \cdot \mathbf{u}_{\mathbf{1}}{ }^{\prime}\right)\right|}=r^{2} d \Omega
$$

where $\mathfrak{u}_{1}{ }^{\prime}\left(x_{\alpha}{ }^{\prime}\right)$ is the radial unit vector at the source point. The radial component of the noise power flow density received by the antenna located on the surface of the Earth may be given as follows, with the aid of equation (49):

$$
\bar{p}_{r}\left(\omega, x_{\alpha}\right)=\mathbf{p}\left(\omega, x_{\alpha}\right) \cdot \mathbf{u}_{1}\left(x_{\alpha}\right)=\frac{k_{0} \Delta f}{2 \lambda^{2}} \int_{h_{0}}^{h_{1}} \int_{\Omega_{a}} y\left(x_{\alpha}, x_{\alpha}^{\prime}\right) \cdot\left[\frac{k T_{0} X Z}{1+Z^{2}}\right] \Gamma d \Omega_{a} d h,
$$


where

$$
y\left(x_{\alpha}, x_{\alpha}{ }^{\prime}\right)=\frac{\mathbf{n}\left(x_{\alpha}, x_{\alpha}{ }^{\prime}\right) \cdot \mathbf{u}_{1}\left(x_{\alpha}\right)}{\left|\mathbf{n}\left(x_{\alpha}, x_{\alpha}\right) \cdot \mathbf{u}_{1}{ }^{\prime}\left(x_{\alpha}{ }^{\prime}\right)\right|}=\frac{\cos \psi_{0}}{\cos \psi_{0}{ }^{\prime}}
$$

and $\Omega_{a}$ is the solid angle representing the beam area of the receiving antenna and $r=a+h$ is used in the derivation. The angles $\psi_{0}$ and $\psi_{0}^{\prime}$ appearing in equation 55 are those between $\mathbf{n}$ and $\mathbf{u}_{1}$ and between $\mathbf{n}$ and $\mathbf{u}_{1}^{\prime}$ respectively, and they are related geometrically as is shown in Fig. $\mathrm{I}$.

If an antenna of sufficiently small beam area is used for measurement, some approximation can be made in equation (54). That is to say, if $\Omega_{\alpha}$ is sufficiently small, then the unit vector $\mathbf{n}\left(x_{\alpha}, x_{\alpha}{ }^{\prime}\right)$ may be considered as a constant vector within the solid angle $\Omega_{a}$, and may be replaced by $\overline{\mathbf{n}}\left(x_{\alpha}, q_{\alpha}\right)$, where $q_{\alpha}$ is the representative source point lying on the axis of $\Omega_{a}$ and the factor $y$ given in equation $(55)$ becomes independent of the source point $x_{\alpha}{ }^{\prime}$ also. Therefore from equations (52) and (54), the expression for the avalable noise power at the receiving antenna is

$$
P_{r}(\omega)=\frac{k_{0} \Delta f}{2} \bar{y} \int_{h_{n}}^{h_{1}}\left[\begin{array}{c}
l T_{0} X Z \\
1+Z^{2}
\end{array}\right] \bar{\Gamma} d h
$$

where

$$
\bar{y}=\frac{\overline{\mathbf{n}} \cdot \mathbf{u}_{1}\left(x_{x}\right)}{\overline{\mathbf{n}} \cdot \mathbf{u}_{1}^{\prime}\left(q_{z}\right)}
$$

and

$$
\bar{\Gamma}=\left(1-\bar{n}_{1}^{2}\right) l_{11}(h)+\left(1-\bar{n}_{2}^{2}\right) l_{22}(h)+\left(1-\bar{n}_{3}^{2}\right) l_{33}(h)-2 \bar{n}_{1} \bar{n}_{2} l_{12}(h) .
$$

It is observed that for the case of a vertical incident measurement, $\mathbf{u}_{1}=\mathbf{u}_{1}{ }^{\prime}$, $\tilde{n}_{1}=1$ and $\bar{n}_{2}=\bar{n}_{3}=0$, so that $\Gamma_{0}=l_{22}+l_{33}$ and $\bar{y}=1$. Consequently the available thermal noise power at the receiving antenna per unit bandwidth, $P_{0}(f)$, for the case of vertical incident measurement may be given by

$$
P_{0}(f)=\pi \int_{h_{0}}^{h_{1}}\left[\begin{array}{l}
k T_{0} X Z \\
1+Z^{2}
\end{array}\right] \bar{\Gamma}_{0} d h
$$

It is interesting to note that for a special case $Y=0$ (corresponding to the absence of a geomagnetic field), $l_{22}=l_{33}=1$ and $\Gamma_{0}=2$. Furthermore, if $Z^{2} \ll 1$, then equation (59) is reduced essentially to the same form as that used by many workers (Pawsey et al., 1951; Gardner, 1954; Dowden, 1960; Litrle et al., 1961 ; Davis, 1960; WHTTEHEAD, 1959).

\section{Concluding Remarks}

The attention has been focused in the present study on the effect of colliding electrons under the assumption that the effect of the motion of ions in the region of the ionosphere of interest is negligible.

The general expressions derived for $w_{0}$, the thermal noise power generated per unit volume, per unit bandwidth, from any given source region $V_{s}$ of the ionosphere, and for $P_{0}(f)$, the available thermal power per unit bandwidth received at the detecting antenna due to the radiation from $V_{k}$, are valid for all frequency ranges and for most regions of interest in the ionosphere, i.e. Where the electron collision process plays a major role. Once $V_{s}$ is specified, the profiles of $T_{0}(h), N_{0}(h)$ and 
$v(h)$ obtained from the experimental observations (NICOLET, 1959; Thomas, 1959; KaNE, 1960a, 1960b; ATAEv, 1959; SchlaPP, 1959) can be used for the evaluation of $w_{0}$ and $P_{0}(f)$. Thus the detailed information with regard to the spectral distribution of the thermal energy radiated from the ionosphere can be obtained with the aid of a numerical integration of the expressions derived in the present paper.

It is indeed desirable that the present theory be tested and verified with some sort of experimental observation, e.g. a laboratory experiment. In other words, if the ionospheric plasma condition can be realistically represented with a laboratory experiment, then it will permit a study of the characteristics of thermal radiation in great detail and a test of the soundness of the present theory.

It should be pointed out that the present analysis may not be as rigorous as a microscopic treatment using the Boltzmann transport equation with the proper collision integral. However, this method of analysis does offer a simple and direct way of analyzing the thermal radiation from an anisotropic ionized medium and its radiation characteristics.

Acknowledgements - The author is very grateful to Professor J. E. Rowe for his contributions and for reading the manuscript. The work was supported by the National Aeronautics and Space Administration under Grant No. NsG 696.

\section{REFERENCES}

Ataev O. M.

Bland-Lapierse A. and Fontet R.

DAVIS K.

DOWDEN R. L.

GARDNER F. F.

HAUS H. A.

KANE J. A.

KraUS J. D.

LitTle C. G., Lerfald G. M. and

Parthasarathy $R$.

Morgan M. G.

NICOLET M.

Pawsey J. L., MoCready L. L. and GaRDNER F. F.

RYTOV S. M.

SCHLAPP D. M.

Stratton J. A.

Thomas J. 0.

Vanwormhoudt M. and Haus $H$. A. WHITEHEAD J. D.
1959 Radio Engng Electron., Wash. 4, 37.

1953 Théorie des Fonctions Aléatories, p. 382, Masson, Paris.

$1960 \quad J$. Geophys. Res. 65, 2285.

1960 J. Atmosph. Terr. Phys. 18, 8.

$1954 J$ J. Atmosph. Terr. Phys. 5, 298.

1961 J. Appl. Phys. $32,493$.

$1960 b \quad J$. Geophys. Res. 64, 133.

1950 Antennas, Chapt. III., p. 41. McGrawHill, New York.

1961 J. Atmosph. Terr. Phys. 28, 275.

1959 Proc. Instn. Radio Engrs. Aust. 47, 131.

1959 Proc. Instn. Radio Engrs. Aust. 47, 142.

1951 J. Atmosph. Terr. Phys. 1, 261.

1959

Theory of Electrical Fluctuation and Thermal Radiation, Transl. Electronic Research Directorate, U.S. Air Force, Bedford, Mass.

1959 J. Atmosph. Terr. Phys. 16, 340.

1941 Electromagnetic Theory, p. 429. McGrawHill, New York.

1959 Proc. Instn. Radio Engrs. Aust. 47, 162.

$1962 J$. Appl. Phys. 33, 2572.

1959 J. Atmosph. Terr. Phys. 16, 283. 